\title{
Barrier Solutions of Elliptic Differential Equations in Musielak-Orlicz-Sobolev Spaces
}

\author{
Ge Dong $\mathbb{B}^{1}$ and Xiaochun Fang $\mathbb{D}^{2,3}$ \\ ${ }^{1}$ Department of Arts and Science Teaching, Shanghai University of Medicine and Health Sciences, Shanghai 201318, China \\ ${ }^{2}$ Department of Mathematics, Tongji University, Shanghai 200092, China \\ ${ }^{3}$ North Caucasus Center for Mathematical Research, 362025 Vladikavkaz, Russia
}

Correspondence should be addressed to Xiaochun Fang; xfang@tongji.edu.cn

Received 27 March 2021; Accepted 21 August 2021; Published 7 October 2021

Academic Editor: Pasquale Vetro

Copyright (C) 2021 Ge Dong and Xiaochun Fang. This is an open access article distributed under the Creative Commons Attribution License, which permits unrestricted use, distribution, and reproduction in any medium, provided the original work is properly cited.

In this paper, we study the solution set of the following Dirichlet boundary equation: $-\operatorname{div}\left(a_{1}(x, u, D u)\right)+a_{0}(x, u)=f(x, u, D u)$ in Musielak-Orlicz-Sobolev spaces, where $a_{1}: \Omega \times \mathbb{R} \times \mathbb{R}^{N} \longrightarrow \mathbb{R}^{N}, a_{0}: \Omega \times \mathbb{R} \longrightarrow \mathbb{R}$, and $f: \Omega \times \mathbb{R} \times \mathbb{R}^{N} \longrightarrow \mathbb{R}$ are all Carathéodory functions. Both $a_{1}$ and $f$ depend on the solution $u$ and its gradient $D u$. By using a linear functional analysis method, we provide sufficient conditions which ensure that the solution set of the equation is nonempty, and it possesses a greatest element and a smallest element with respect to the ordering " $\leq$," which are called barrier solutions.

\section{Introduction}

Let $\Omega \subset \mathbb{R}^{N}$ be a bounded domain with Lipschitz boundary. Fan [1] established a sub-supersolution method for the following differential equations of divergence form:

$$
-\operatorname{div}\left(a_{1}(x, D u)\right)+a_{0}(x, u)=f(x, u),
$$

in $\Omega$ coupled with Neumann or Dirichlet boundary condition in reflexive Musielak-Orlicz-Sobolev spaces $W_{0}^{1}$ $L_{\Phi}(\Omega)$ and obtained the existence of greatest and smallest solutions within the order interval $[\underline{u}, \bar{u}]$, where $\underline{u}:=\max$ $\left\{\underline{u}_{1}, \underline{u}_{2}, \cdots, \underline{u}_{k}\right\}, \bar{u}:=\min \left\{\bar{u}_{1}, \bar{u}_{2}, \cdots, \bar{u}_{m}\right\}$, and $\underline{u}_{1}, \underline{u}_{2}, \cdots, \underline{u}_{k}$ and $\bar{u}_{1}, \bar{u}_{2}, \cdots, \bar{u}_{m}$ are subsolutions and supersolutions, respectively. Dong and Fang [2] studied the existence of weak solutions for the following equations:

$$
-\operatorname{div}\left(a_{1}(x, D u)\right)+a_{0}(x, u)=f(x, u, D u)
$$

in $\Omega$ coupled with Neumann or Dirichlet boundary condition in separable Musielak-Orlicz-Sobolev spaces and obtained the existence of greatest and smallest solutions within the order interval $[\underline{u}, \bar{u}]$. Carl and Le [3] established the enclosure of solutions, i.e., $[\underline{u}, \bar{u}]$, for multivalued elliptic variational inequalities by using the sub-supersolution method in Sobolev spaces. However, Carl [4] pointed out that there may exist some more solutions of some elliptic equations which are not within the corresponding interval $[\underline{u}, \bar{u}]$. For example, the solution set $\delta$ of the following Dirichlet boundary value problem

$$
\begin{cases}-\Delta u-\lambda u=0, & \text { in } \Omega, \\ u=0, & \text { on } \partial \Omega,\end{cases}
$$

is given by $\mathcal{S}=\left\{u=c \varphi_{1}: c \in \mathbb{R}\right\}$, where $\varphi_{1}$ is the eigenfunction belonging to $\lambda_{1}$, and $\lambda_{1}>0$ is the first eigenvalue of the negative Laplacian $-\Delta$ (see [4]). Then, $\delta \neq \varnothing$ neither is norm bounded nor has the extreme solutions. Therefore, Carl [4] provided some sufficient conditions which ensure that the solution set of an elliptic variational inequality possesses a greatest element $u^{*}$ and a smallest element $u_{*}$ with respect to the natural partial ordering of functions in Sobolev spaces. Here, $u^{*}$ and $u_{*}$ are called barrier solutions (see [4]). In [5], Chipot et al. 
established the existence of the minimal solution to some elliptic variational inequalities in Sobolev spaces.

It is well known that different classes of differential equations correspond to different function space settings. The Musielak-Orlicz-Sobolev spaces are the extensions of Sobolev spaces, variable exponent Sobolev spaces, and Orlicz-Sobolev spaces. Gwiazda et al. [6] proved the existence of renormalized solutions to the following nonlinear elliptic equations:

$$
-\operatorname{div} A(x, D u)=f
$$

in $\Omega$ coupled with Dirichlet boundary condition in MusielakOrlicz-Sobolev spaces, where $A: \Omega \times \mathbb{R}^{N} \longrightarrow \mathbb{R}^{N}$ is a Carathéodory function and $f \in L^{1}(\Omega)$. The growth of $A$ is controlled by Musielak-Orlicz function $\Phi$ :

$$
A(x, \xi) \xi \geq c_{A}(\Phi(x,|\xi|)+\bar{\Phi}(x,|A(x, \xi)|))
$$

for all $x \in \Omega$ and $\xi \in \mathbb{R}^{N}$, where $\bar{\Phi}$ is a complementary function to $\Phi$, the constant $c_{A} \in(0,1]$. Although they did not require $\Phi \in \Delta_{2}$ nor $\bar{\Phi} \in \Delta_{2}$, condition (5) is stronger than the growth condition (3.1) and the coercive condition (3.2) in [2]. In [7], Chlebicka and Karppinen studied removable sets for elliptic equations of the form

$$
-\operatorname{div} A(x, D u)=0
$$

in $\Omega$, where $A$ satisfies the nonstandard growth condition and the coercivity condition expressed by means of a Musielak-Orlicz function.

The purpose of this paper is to provide sufficient conditions which ensure that the solution set $\mathcal{S}$ of the following Dirichlet boundary problem

$$
\begin{cases}-\operatorname{div}\left(a_{1}(x, u, D u)\right)+a_{0}(x, u)=f(x, u, D u), & \text { in } \Omega, \\ u=0, & \text { on } \partial \Omega,\end{cases}
$$

is nonempty and possesses barrier solutions in MusielakOrlicz-Sobolev spaces, where $a_{1}: \Omega \times \mathbb{R} \times \mathbb{R}^{N} \longrightarrow \mathbb{R}^{N}, a_{0}$ : $\Omega \times \mathbb{R} \longrightarrow \mathbb{R}$, and $f: \Omega \times \mathbb{R} \times \mathbb{R}^{N} \longrightarrow \mathbb{R}$ are Carathéodory functions. Both $a_{1}$ and $f$ depend on the solution $u$ and its gradient $\mathrm{Du}$.

This paper is organized as follows: Section 2 contains some preliminaries and some technical lemmas which will be needed. In Section 3, we first give the sufficient conditions which ensure the existence of weak solutions for (7) in Musielak-Orlicz-Sobolev spaces. Some examples are given for the application of our results. We point that (5) is strong. Then, we provide some sufficient conditions which ensure that the solution set of (7) is bounded, compact, and direct. Finally, we provide some sufficient conditions which ensure that the solution set of (7) possesses barrier solutions in Musielak-Orlicz-Sobolev spaces.

We refer to some results in variable exponent Sobolev or Orlicz-Sobolev spaces [8-22].

\section{Preliminaries}

Now, we list briefly some definitions and facts about MusielakOrlicz-Sobolev spaces; for more details, see [1, 2, 23-25].

$\Phi: \Omega \times \mathbb{R}_{+}=[0,+\infty) \longrightarrow \mathbb{R}_{+}$is called a generalized $N$ function (i.e., a Musielak-Orlicz function), denoted by $\Phi \in$ $N(\Omega)$, if it satisfies the following conditions:

(i) $\Phi(x, u)$ is an $N$-function of the variable $u \geq 0$ for every $x \in \Omega$, i.e., it is a convex, nondecreasing, and continuous function of $u$ such that $\Phi(x, 0)=0$, $\Phi(x, u)>0$ for $u>0$, and there hold the conditions

$$
\begin{aligned}
& \lim _{u \longrightarrow 0^{+}} \sup _{x \in \Omega} \frac{\Phi(x, u)}{u}=0, \\
& \lim _{u \longrightarrow+\infty} \inf _{x \in \Omega} \frac{\Phi(x, u)}{u}=+\infty
\end{aligned}
$$

(ii) $\Phi(x, u)$ is a measurable function of $x$ for all $u \geq 0$

Equivalently, for all $x \in \Omega$ and all $u \geq 0, \Phi$ admits the representation $\Phi(x, u)=\int_{0}^{u} \varphi(x, \tau) d \tau$, where $\varphi(x, t)$ is the right-hand derivative of $\Phi(x, \cdot)$ at $t$, for a fixed $x \in \Omega$ and all $t \geq 0$. Then, for every $x \in \Omega, \varphi(x, t)$ is a right-continuous and nondecreasing function of $t \geq 0, \varphi(x, 0)=0, \varphi(x, t)>0$ for $t>0$, and $\varphi(x, t) \longrightarrow+\infty$ as $t \longrightarrow+\infty$ for every $x \in \Omega$. The complementary function $\bar{\Phi}$ to $\Phi$ is defined as follows:

$$
\bar{\Phi}(x, v)=\sup _{u \geq 0}\{u v-\Phi(x, u)\} \text { for all } v \geq 0, x \in \Omega
$$

Then, $\bar{\Phi} \in N(\Omega)$ and $\Phi$ is also the complementary function to $\bar{\Phi}$. Equivalently, $\bar{\Phi}$ admits the representation $\bar{\Phi}(x, v)=\int_{0}^{v} \phi(x, \sigma) d \sigma$, where $\phi$ is given by $\phi(x, \sigma)=\sup$ $\{\tau: \varphi(x, \tau) \leq \sigma\}$, for all $x \in \Omega$.

Moreover, one has the Young inequality: $u v \leq \Phi(x, u)+$ $\bar{\Phi}(x, v)$, for all $u, v \geq 0, x \in \Omega$.

$\Phi$ is said to satisfy the $\Delta_{2}$ condition ( $\Phi \in \Delta_{2}$, for short), if there exists a positive constant $K>1$ and a nonnegative function $h \in L^{1}(\Omega)$ such that $\Phi(x, 2 u) \leq K \Phi(x, u)+h(x)$, for all $u \geq 0$ and a.e. $x \in \Omega$.

$\Phi$ is called locally integrable, if $\int_{\Omega} \Phi(x, u) d x<\infty$ for every $u>0$.

The following assumptions will be used.

$\left(\Phi_{1}\right) \inf _{x \in \Omega} \Phi(x, 1)=c_{1}>0$.

$\left(\Phi_{2}\right)$ For every $t_{0}>0$, there exists a $c=c\left(t_{0}\right)>0$ such that

$$
\begin{aligned}
& \frac{\Phi(x, t)}{t} \geq c, \\
& \frac{\bar{\Phi}(x, t)}{t} \geq c,
\end{aligned}
$$

for all $t \geq t_{0}$, and all $x \in \Omega$.

Clearly, (10) implies $\left(\Phi_{1}\right)$. 
Let $\Phi \in N(\Omega)$. We denote by $L^{0}(\Omega)$ the set of all (equivalence classes of) Lebesgue measurable functions from $\Omega$ to $\mathbb{R}$. The Musielak-Orlicz space (i.e., the generalized Orlicz space) $L_{\Phi}(\Omega)$ is defined by

$$
\begin{aligned}
L_{\Phi}(\Omega) & =\left\{u \in L^{0}(\Omega): \rho_{\Phi}\left(\frac{u}{\lambda}\right)\right. \\
& \left.:=\int_{\Omega} \Phi\left(x, \frac{|u(x)|}{\lambda}\right) d x<\infty, \text { for some } \lambda>0\right\},
\end{aligned}
$$

with the (Luxemburg) norm

$$
\|u\|_{\Phi}=\inf \left\{\lambda>0: \rho_{\Phi}\left(\frac{u}{\lambda}\right) \leq 1\right\} .
$$

Moreover, the set

$$
K_{\Phi}(\Omega)=\left\{u \in L^{0}(\Omega): \rho_{\Phi}(u)<+\infty\right\}
$$

will be called the Musielak-Orlicz class (i.e., the generalized Orlicz class). A function $u \in L^{0}(\Omega)$ will be called a finite element of $L_{\Phi}(\Omega)$, if $\lambda u \in K_{\Phi}(\Omega)$ for every $\lambda>0$. The space of all finite elements of $L^{0}(\Omega)$ will be denoted by $E_{\Phi}(\Omega)$. Then, $K_{\Phi}(\Omega)$ is a convex subset of $L_{\Phi}(\Omega), L_{\Phi}(\Omega)$ is the smallest vector subspace of $L^{0}(\Omega)$ containing $K_{\Phi}(\Omega)$, and $E_{\Phi}(\Omega)$ is the largest vector subspace of $L^{0}(\Omega)$ contained in $K_{\Phi}(\Omega)$.

If $\Phi$ is locally integrable, then $E_{\Phi}(\Omega)$ is a separable space, and $E_{\Phi}(\Omega)=K_{\Phi}(\Omega)=L_{\Phi}(\Omega)$ if and only if $\Phi \in \Delta_{2}$.

If $\Phi$ is locally integrable and satisfy (10), then $\left(E_{\Phi}(\Omega)\right)^{*}=L_{\bar{\Phi}}(\Omega)$. Moreover, if $\bar{\Phi}$ is also locally integrable satisfying (11), and $\Phi, \bar{\Phi} \in \Delta_{2}$, then $L_{\Phi}(\Omega)$ is reflexive. by

The Musielak-Orlicz-Sobolev space $W^{1} L_{\Phi}(\Omega)$ is defined

$$
W^{1} L_{\Phi}(\Omega)=\left\{u \in L_{\Phi}(\Omega): \forall|\alpha| \leq 1, D^{\alpha} u \in L_{\Phi}(\Omega)\right\},
$$

where $\alpha=\left(\alpha_{1}, \cdots, \alpha_{N}\right)$ with nonnegative integers $\alpha_{i}, i=1, \cdots$, $N,|\alpha|=\left|\alpha_{1}\right|+\left|\alpha_{2}\right|+\cdots+\left|\alpha_{N}\right|$ and $D^{\alpha} u$ denote the distributional derivatives.

Let

$$
\begin{aligned}
\rho_{\Phi}(u) & =\sum_{|\alpha| \leq 1} \int_{\Omega} \Phi\left(x,\left|D^{\alpha} u(x)\right|\right) d x, \\
\|u\|_{\Phi, \Omega} & =\inf \left\{\lambda>0: \rho_{\Phi}\left(\frac{u}{\lambda}\right) \leq 1\right\},
\end{aligned}
$$

for $u \in W^{1} L_{\Phi}(\Omega)$. Then, $\|u\|_{\Phi, \Omega}$ is a norm on $W^{1} L_{\Phi}(\Omega)$. The pair $\left(W^{1} L_{\Phi}(\Omega),\|u\|_{\Phi, \Omega}\right)$ is a Banach space if $\Phi$ is locally integrable and satisfies $\left(\Phi_{1}\right)$.

If $\Phi(x, u)=\Phi(u)$ is independent of $x$, then $W^{1} L_{\Phi}(\Omega)$ becomes the Orlicz-Sobolev space. Let $p \in C_{+}(\bar{\Omega})$, where $C_{+}(\bar{\Omega})=\{p: \bar{\Omega} \longrightarrow \mathbb{R}$ is continuous, $p(x)>1, \forall x \in \bar{\Omega}\}$. If
$\Phi(x,|u|)=|u|^{p(x)}$, then $W^{1} L_{\Phi}(\Omega)$ becomes the variable exponent Sobolev space

$$
W^{1, p(x)}(\Omega)=\left\{u \in L^{p(x)}(\Omega) \mid D u \in\left(L^{p(x)}(\Omega)\right)^{N}\right\},
$$

where $L^{p(x)}(\Omega)=\left\{\left.u \in L^{0}(\Omega)\left|\int_{\Omega}\right| u(x)\right|^{p(x)} d x<+\infty\right\} . L^{p(x)}(\Omega)$ is equipped with the Luxemburg norm

$$
\|u\|_{L^{p(x)}(\Omega)}=\inf \left\{\lambda>0: \int_{\Omega}\left|\frac{u(x)}{\lambda}\right|^{p(x)} d x \leq 1\right\}\left(u \in L^{p(x)}(\Omega)\right),
$$

and $W^{1} L_{\Phi}(\Omega)$ is equipped with the norm

$$
\|u\|_{W^{1, p(x)}(\Omega)}=\|u\|_{L^{p(x)}(\Omega)}+\|\mid D u\|_{L^{p(x)}(\Omega)}\left(u \in W^{1, p(x)}(\Omega)\right) .
$$

It is easy to see that

$$
W^{1} L_{\Phi}(\Omega)=\left\{u \in L_{\Phi}(\Omega):|D u| \in L_{\Phi}(\Omega)\right\} .
$$

Denote $\|D u\|_{\Phi}=\|\mid D u\|_{\Phi}$ and $\|u\|_{1, \Phi}=\|u\|_{\Phi}+\|D u\|_{\Phi}$. Clearly, $\|u\|_{1, \Phi}$ is equivalent to $\|u\|_{\Phi, \Omega}$.

The space $W^{1} L_{\Phi}(\Omega)$ can be identified with a subspace of the product of $N+1$ copies of $L_{\Phi}(\Omega)$. Denote the product by $\prod L_{\Phi}$. The subspace is $\sigma\left(\prod L_{\Phi}, \prod E_{\bar{\Phi}}\right)$ closed. Let $W_{0}^{1} L_{\Phi}(\Omega)$ be the $\sigma\left(\prod L_{\Phi}, \prod E_{\bar{\Phi}}\right)$ closure of the Schwartz space $\mathscr{D}(\Omega)$ in $W^{1} L_{\Phi}(\Omega)$.

The space $W^{1} E_{\Phi}(\Omega)$ can be identified with a subspace of the product of $N+1$ copies of $E_{\Phi}(\Omega)$. Denote the product by $\prod E_{\Phi}$. Let $W_{0}^{1} E_{\Phi}(\Omega)$ be the (norm) closure of $\mathscr{D}(\Omega)$ in $W^{1} L_{\Phi}(\Omega)$.

Lemma 1 (see [23], Lemma 14.4). Let $\Phi \in N(\Omega), \bar{\Phi}$ be a complementary function to $\Phi$, and $\Phi^{-1}, \bar{\Phi}^{-1}$ be the inverse functions to $\Phi, \bar{\Phi}$, respectively. Then,

$$
u \leq \Phi^{-1}(x, u) \bar{\Phi}^{-1}(x, u) \leq 2 u, \text { for every } u \geq 0, x \in \Omega
$$

By Lemma 1, we can deduce the following lemma.

Lemma 2. Let $\Phi \in N(\Omega), \bar{\Phi}$ be a complementary function to $\Phi$ and $\Phi^{-1}, \bar{\Phi}^{-1}$ be the inverse functions to $\Phi, \bar{\Phi}$, respectively. Then,

$\frac{\Phi(x, u)}{u} \leq \bar{\Phi}^{-1}(x, \Phi(x, u)) \leq \frac{2 \Phi(x, u)}{u}$, for every $u>0, x \in \Omega$. 
Lemma 3 (see [26], Lemma 2.1). If $u \in W^{1} L_{\Phi}(\Omega)$, then $u^{+}$, $u^{-} \in W^{1} L_{\Phi}(\Omega)$ and

$$
\begin{gathered}
D u^{+}= \begin{cases}D u, & \text { if } u>0, \\
0, & \text { if } u \leq 0,\end{cases} \\
D u^{-}= \begin{cases}0, & \text { if } u \geq 0, \\
-D u, & \text { if } u<0 .\end{cases}
\end{gathered}
$$

Here, $u^{+}=\max \{u, 0\}, u^{-}=-\min \{u, 0\}$.

\section{Existence Theorems}

Let $\Omega \subset \mathbb{R}^{N}$ be a bounded domain with Lipschitz boundary. Let $\Phi \in N(\Omega), \bar{\Phi}$ be a complementary function to $\Phi$ with $\bar{\Phi} \in \Delta_{2}, \Phi$ and $\bar{\Phi}$ be locally integrable. Suppose that the embedding $W_{0}^{1} L_{\Phi}(\Omega)^{\hookrightarrow} L_{\Phi}(\Omega)$ is compact.

For $u, v \in L^{0}(\Omega)$, we use the standard notations: $u \wedge v=$ $\min \{u, v\}, u \vee v=\max \{u, v\}, u^{+}:=u \vee 0, u^{-}:=-u \wedge 0, u \leq v$ $\Leftrightarrow u(x) \leq v(x)$ for a.e. $x \in \Omega$. According to [1] (Remark 3.1), $u \vee v, u \wedge v \in W^{1} L_{\Phi}(\Omega)$, for any $u, v \in W^{1} L_{\Phi}(\Omega)$. Clearly, $u \vee v, u \wedge v \in W_{0}^{1} L_{\Phi}(\Omega)$, if $u, v \in W_{0}^{1} L_{\Phi}(\Omega)$.

Let $a_{1}: \Omega \times \mathbb{R} \times \mathbb{R}^{\mathbb{N}} \longrightarrow \mathbb{R}^{\mathbb{N}}$ be a Carathéodory function satisfying the following conditions:

$\left(A_{1}\right)$ For a.e. $x \in \Omega$, all $s \in \mathbb{R}$ and all $\xi, \eta \in \mathbb{R}^{N}$,

$$
\begin{gathered}
\left|a_{1}(x, s, \xi)\right| \leq g_{1}(x)+b_{1} \bar{\Phi}^{-1}\left(x, \Phi\left(x, k_{1}|s|\right)\right) \\
+b_{2} \bar{\Phi}^{-1}\left(x, \Phi\left(x, k_{2}|\xi|\right)\right), \\
a_{1}(x, s, \xi) \xi \geq b_{3} \Phi(x,|\xi|)-g_{2}(x), \\
{\left[a_{1}(x, s, \xi)-a_{1}(x, s, \eta)\right](\xi-\eta)>0, \xi \neq \eta,}
\end{gathered}
$$

where $b_{1}, b_{2}, b_{3}, k_{1}, k_{2}>0, g_{1} \in E_{\bar{\Phi}}(\Omega), g_{1} \geq 0, g_{2} \in L^{1}(\Omega)$, and $g_{2} \geq 0$.

Let $a_{0}: \Omega \times \mathbb{R} \longrightarrow \mathbb{R}$ be a Carathéodory function satisfying the following conditions:

$\left(A_{0}\right)$ For a.e. $x \in \Omega$ and all $s \in \mathbb{R}$,

$$
\begin{aligned}
& \left|a_{0}(x, s)\right| \leq g_{1}(x)+b_{1} \bar{\Phi}^{-1}\left(x, \Phi\left(x, k_{1}|s|\right)\right), \\
& a_{0}(x, s) s \geq b_{3} \Phi(x,|s|)-g_{2}(x),
\end{aligned}
$$

where $b_{1}, b_{3}, k_{1}>0, g_{1} \in E_{\bar{\Phi}}(\Omega), \quad g_{1} \geq 0, g_{2} \in L^{1}(\Omega)$, and $g_{2} \geq 0$.

Let $f: \Omega \times \mathbb{R} \times \mathbb{R}^{N} \longrightarrow \mathbb{R}$ be a Carathéodory function satisfying the following growth condition:

$(F)$ For a.e. $x \in \Omega$, all $s \in \mathbb{R}$, and all $\xi \in \mathbb{R}^{N}$,

$$
|f(x, s, \xi)| \leq q(x)+b_{4} \bar{\Phi}^{-1}(x, \Phi(x,|s|))+b_{5} \bar{\Phi}^{-1}(x, \Phi(x,|\xi|)),
$$

where $q \in E_{\bar{\Phi}}(\Omega), b_{4}, b_{5}>0$. Denote by $F$ the Nemytskii operator associated to $f$, that is,

$$
F(u)(x)=f(x, u(x), D u(x)), \text { for } x \in \Omega \text {. }
$$

Consider the following Dirichlet boundary value problem: $\begin{cases}-\operatorname{div}\left(a_{1}(x, u, D u)\right)+a_{0}(x, u)=f(x, u, D u), & \text { in } \Omega, \\ u=0, & \text { on } \partial \Omega\end{cases}$

A function $u$ is called a weak solution of (31) if $u \in$ $W_{0}^{1} L_{\Phi}(\Omega), F(u) \in L_{\bar{\Phi}}(\Omega)$, and $u$ satisfies the equation

$$
\begin{aligned}
\int_{\Omega} a_{1}(x, u, D u) D v d x+\int_{\Omega} a_{0}(x, u) v d x \\
\quad=\int_{\Omega} f(x, u, D u) v d x, \text { for all } v \in W_{0}^{1} L_{\Phi}(\Omega) .
\end{aligned}
$$

A function $u$ is called a subsolution (resp., supersolution) of (31) if $u \in W_{0}^{1} L_{\Phi}(\Omega), F(u) \in L_{\bar{\Phi}}(\Omega)$, and (32) holds with "=" replaced by " $\leq$ " (resp., " $\geq$ ") for every nonnegative functions $v$ in $W_{0}^{1} L_{\Phi}(\Omega)$ (see [1]).

Theorem 4. Let $\left(A_{1}\right),\left(A_{0}\right)$, and $(F)$ hold. If

$$
2 b_{4}+b_{5}<b_{3}
$$

then there exists at least one weak solution of (31).

Proof. Denote $V=W_{0}^{1} E_{\Phi}(\Omega)$. Define $\Gamma: V \longrightarrow V^{*}$,

$$
\begin{aligned}
(\Gamma u, v):= & \int_{\Omega} a_{1}(x, u, D u) D v d x+\int_{\Omega} a_{0}(x, u) v d x \\
& -\int_{\Omega} F(u) v d x
\end{aligned}
$$

$\forall v \in V$. Then, $\Gamma$ is well defined.

Since $V$ is separable, there is a sequence $\left\{w_{n}\right\}_{n=1}^{\infty} \subset \mathscr{D}(\Omega)$ such that $\left\{w_{n}\right\}_{n=1}^{\infty}$ is dense in $V$. Let $V_{m}=\operatorname{span}\left\{w_{1}, \cdots, w_{m}\right\}$ and consider $\left.\Gamma\right|_{V_{m}}$.

Similar to the proof of Theorem 3.1 in [27], $\left.u \longrightarrow \Gamma\right|_{V_{m}}$ $u: V_{m} \longrightarrow V_{m}^{*}$ is continuous.

In view of (29), for every $u \in V$, we have

$$
\begin{aligned}
\left|\int_{\Omega} F(u) u d x\right| \leq & C_{1}^{*}\|q\|_{\bar{\Phi}}\|u\|_{1, \Phi}+\left(2 b_{4}+b_{5}\right) \int_{\Omega} \Phi(x,|u|) d x \\
& +b_{5} \int_{\Omega} \Phi(x,|D u|) d x
\end{aligned}
$$

for some constant $C_{1}^{*}>0$. 
Combining (25), (28), (33), and (35), we obtain

$$
\begin{aligned}
(\Gamma u, u) \geq & \left(b_{3}-b_{5}\right) \int_{\Omega} \Phi(x,|D u|) d x \\
& +\left(b_{3}-2 b_{4}-b_{5}\right) \int_{\Omega} \Phi(x,|u|) d x-C-C_{1}^{*}\|q\|_{\bar{\Phi}}\|u\|_{1, \Phi} \\
\geq & \left(b_{3}-b_{5}\right) \int_{\Omega} \Phi(x,|D u|) d x-C-C_{1}^{*}\|q\|_{\bar{\Phi}}\|u\|_{1, \Phi},
\end{aligned}
$$

for all $u \in V$, and some constant $C>0$ independent of $u$.

Consequently,

$$
\begin{aligned}
\frac{(\Gamma u, u)}{\|u\|_{1, \Phi}} \geq \frac{\left(b_{3}-b_{5}\right) \int_{\Omega} \Phi(x,|D u|) d x}{\|u\|_{1, \Phi}}-\frac{C}{\|u\|_{1, \Phi}}-C_{1}^{*}\|q\|_{\Phi} \\
\geq\left(b_{3}-b_{5}\right) \frac{\int_{\Omega} \Phi(x,|D u|) d x}{C_{2}^{*}\|D u\|_{L^{1}(\Omega)}}-\frac{C}{\|u\|_{1, \Phi}}-C_{1}^{*}\|q\|_{\Phi},
\end{aligned}
$$

for all $u \in V_{m}$, and some constant $C_{2}^{*}>0$. Using ([2], Lemma $1)$, we have

$$
\frac{(\Gamma u, u)}{\|u\|_{1, \Phi}} \longrightarrow+\infty, \quad \text { as }\|u\|_{1, \Phi} \longrightarrow+\infty
$$

By [27] (Remark 2.1), there exists a Galerkin solution $u_{m} \in V_{m}$ for every $m \in \mathbb{N}$ such that

$$
\left(\Gamma u_{m}, v\right)=0, \quad \forall v \in V_{m} .
$$

Using the density of $\left\{w_{n}\right\}$, we deduce that

$$
\left(\Gamma u_{m}, v\right)=0, \quad \forall v \in V .
$$

Therefore, the sequence $\left\{u_{m}\right\}$ is bounded in $V$. Consequently, there exists $u_{0} \in V$, and we can extract a subsequence $\left\{u_{n}\right\}$ of $\left\{u_{m}\right\}$ such that

$$
\begin{gathered}
u_{n} \rightarrow u_{0} \text { weakly in } V \text { for } \sigma\left(\prod L_{\Phi}, \prod E_{\bar{\Phi}}\right), \\
u_{n} \longrightarrow u_{0} \text { strongly in } L_{\Phi}(\Omega), \\
u_{n}(x) \longrightarrow u_{0}(x) \text { for a.e. } x \in \Omega,
\end{gathered}
$$

as $n \longrightarrow \infty$. By (24), $\left\{a_{1}\left(x, u_{n}, D u_{n}\right)\right\}$ is bounded in $\left(L_{\bar{\Phi}}(\Omega)\right)^{N}$, and $\left\{a_{0}\left(x, u_{n}\right)\right\}$ and $\left\{f\left(x, u_{n}, D u_{n}\right)\right\}$ are bounded in $L_{\bar{\Phi}}(\Omega)$. Thanks to (42),

$$
\begin{array}{r}
\int_{\Omega} a_{0}\left(x, u_{n}\right)\left(u_{n}-u_{0}\right) d x \longrightarrow 0, \\
\int_{\Omega} f\left(x, u_{n}, D u_{n}\right)\left(u_{n}-u_{0}\right) d x \longrightarrow 0,
\end{array}
$$

as $n \longrightarrow \infty$. It follows that

$$
\int_{\Omega} a_{1}\left(x, u_{n}, D u_{n}\right)\left(D u_{n}-D u_{0}\right) d x \longrightarrow 0,
$$

as $n \longrightarrow \infty$.

Therefore, similar to [27], we can deduce that there exists a subsequence of $\left\{u_{n}\right\}$ still denoted by $\left\{u_{n}\right\}$ such that

$$
D u_{n}(x) \longrightarrow D u_{0}(x) \text { for a.e. } x \in \Omega \text {, }
$$

as $n \longrightarrow \infty$. Combining (43) and (46), we obtain that

$$
\begin{aligned}
a_{1}\left(x, u_{n}, D u_{n}\right) & \longrightarrow a_{1}\left(x, u_{0}, D u_{0}\right) \text { a.e.in } \Omega, \\
a_{0}\left(x, u_{n}\right) & \longrightarrow a_{0}\left(x, u_{0}\right) \text { a.e.in } \Omega, \\
f\left(x, u_{n}, D u_{n}\right) & \longrightarrow f\left(x, u_{0}, D u_{0}\right) \text { a.e.in } \Omega,
\end{aligned}
$$

as $n \longrightarrow \infty$

Using [25] (Lemma 1) and letting $n \longrightarrow \infty$, passing to a subsequence if necessary, we can deduce from (40) that

$$
\left(\Gamma u_{0}, v\right)=0, \quad \forall v \in V .
$$

By [28] (Lemma 2.5) and [25] (Lemma 7),

$$
\left(\Gamma u_{0}, v\right)=0, \quad \forall v \in W_{0}^{1} L_{\Phi}(\Omega) .
$$

Thus, there exists at least one weak solution $u_{0}$ of (31).

Example 1. Let $\Phi(x,|t|)=e^{p(x)|t|}-p(x)|t|-1$, for $x \in \Omega$ and $t \in \mathbb{R}$, where $p: \Omega \longrightarrow \mathbb{R}$ is a measurable function such that $1<p_{-}:=\inf _{x \in \Omega} p(x) \leq p(x) \leq p_{+}:=\sup _{x \in \Omega} p(x)<+\infty$. Then, $\Phi \notin \Delta_{2}$.

Example 2. Put $a_{1}(x, s, \xi)=a(x, s) \varphi(x,|\xi|) \xi /|\xi|$ if $\xi \in \mathbb{R}^{N} \backslash$ $\{\mathbf{0}\}$ and $a_{1}(x, s, \xi)=\mathbf{0}$ if $\xi=\mathbf{0}$, for a.e. $x \in \Omega$, all $s \in \mathbb{R}$, where $a$ is a Carathéodory function on $\Omega \times \mathbb{R}$ satisfying $\alpha \leq a(x, s)$ $\leq \beta$ with $\alpha>0$ and $\beta>0$ for all $s \in \mathbb{R}, \varphi(x, s)$ is the righthand derivative of $\Phi(x, \cdot)$ at $s$, and $\mathbf{0}$ is the zero vector in $\mathbb{R}^{N}$. Let $a_{0}(x, s)=\alpha \varphi(x,|s|) s /|s|$, and $f(x, s, \xi)=b(x, s) \varphi(x$, $|\xi|)|s|$, for a.e. $x \in \Omega$, all $s \in \mathbb{R}$ and all $\xi \in \mathbb{R}^{N}$, where $b$ is a Carathéodory function on $\Omega \times \mathbb{R}$ satisfying $|b(x, s)| \leq \gamma$ with $0<\gamma<\alpha / 3$. Then, $a_{1}, a_{0}$, and $f$ satisfy conditions $\left(A_{1}\right)$, $\left(A_{0}\right)$, and (29), respectively.

Remark 5. Clearly, condition (5) implies that

$$
\begin{aligned}
& A(x, \xi) \xi \geq c_{A} \Phi(x,|\xi|), \\
& A(x, \xi) \xi \geq c_{A} \bar{\Phi}(x,|A(x, \xi)|),
\end{aligned}
$$

for all $x \in \Omega$ and $\xi \in \mathbb{R}^{N}$. By Lemma 2, we have

$$
\frac{|\xi|}{c_{A}} \geq \frac{\bar{\Phi}(x,|A(x, \xi)|)}{|A(x, \xi)|} \geq \frac{1}{2} \bar{\Phi}^{-1}(x, \bar{\Phi}(x,|A(x, \xi)|)) .
$$


It implies that

$$
\bar{\Phi}(x,|A(x, \xi)|) \leq \Phi\left(x, \frac{2}{c_{A}} \xi\right) .
$$

Consequently,

$$
|A(x, \xi)| \leq \bar{\Phi}^{-1}\left(x, \Phi\left(x, \frac{2}{c_{A}} \xi\right)\right)
$$

Hence, condition (5) is stronger than not only the growth condition (3.1) but also the coercive condition (3.2) in [2].

Let $\mathcal{S}$ be the set of all weak solutions of (31). Under the assumptions of Theorem 4 , the solution set $\mathcal{S}$ of (31) is nonempty.

Theorem 6. Assume that the assumptions of Theorem 4 hold. Then $\mathcal{S}$ is bounded and closed in $W_{0}^{1} L_{\Phi}(\Omega)$. Moreover, $\mathcal{S}$ is compact in $W_{0}^{1} L_{\Phi}(\Omega)$.

Proof. Denote $V=W_{0}^{1} L_{\Phi}(\Omega)$. For $u \in V$, define $\rho(u)=$ $\int_{\Omega}[\Phi(x,|u|)+\Phi(x,|D u|)] d x$, and $\|u\|_{\rho}=\inf \{\lambda>0: \rho(u / \lambda)$ $\leq 1\}$, then $\|u\|_{\rho}$ is a norm of $V$ equivalent to $\|u\|_{1, \Phi}$ (see [1]). Taking $\alpha_{0}=\min \left\{b_{3}-b_{5}, b_{3}-2 b_{4}-b_{5}\right\}$ in the first inequality of (36) and by [2] (Proposition 3.1), there exists $h \in L^{1}(\Omega)$, such that, for every $\varepsilon>0$,

$$
\begin{aligned}
(\Gamma u, u) \geq & \alpha_{0}\left(\|u\|_{\rho}-\varepsilon\right) G\left(\|u\|_{\rho}-\varepsilon\right) \\
& -\alpha_{0}\left(\|u\|_{\rho}-\varepsilon\right) \int_{\Omega}|h(x)| d x-C_{1}-C_{2}\|u\|_{1, \Phi}
\end{aligned}
$$

for all $\forall u \in V$, as $\|u\|_{1, \Phi}$ is large enough, where the constants $C_{1}$ and $C_{2}$ are independent of $u$. Consequently, we can deduce that $\mathcal{S}$ is bounded in $W_{0}^{1} L_{\Phi}(\Omega)$.

Next, we prove that $\mathcal{S}$ is closed. Indeed, assume $\left\{u_{n}\right\}_{n=1}^{\infty} \subset \mathcal{S}$, and $u_{n} \longrightarrow u_{0}$ in $W_{0}^{1} L_{\Phi}(\Omega)$ as $n \longrightarrow \infty$. Since $\mathcal{S}$ is bounded, $\left\{u_{n}\right\}_{n=1}^{\infty}$ is bounded. Therefore, by passing to a subsequence if necessary, we can assume without loss of generality that

$$
u_{n} \rightarrow u_{0} \text { weakly in } V \text { for } \sigma\left(\Pi L_{\Phi}, \Pi E_{\bar{\Phi}}\right)
$$

as $n \longrightarrow \infty$. Similar to the proof of Theorem 4 , we can obtain that $u_{0}$ is a weak solution of (31), i.e., $u_{0} \in \mathcal{S}$. Consequently, $\mathcal{S}$ is closed in $V$.

For any subsequence $\left\{u_{n}\right\}_{n=1}^{\infty}$ in $\mathcal{S},\left\{u_{n}\right\}_{n=1}^{\infty}$ is bounded. Repeating the proof of Theorem 4 , by passing to a subsequence if necessary, we can obtain that $\left\{u_{n}\right\}_{n=1}^{\infty}$ is convergent to $u_{0}$, and $u_{0}$ is a weak solution of (31), i.e., $u_{0} \in \mathcal{S}$. Consequently, $\mathcal{S}$ is sequentially compact. Since $\mathcal{S}$ is closed, $\mathcal{S}$ is compact.
Definition 7 ([4], Definition 2.1$)$. Let $(\mathscr{P}, \leq)$ be a partially ordered set. A subset $\mathscr{C}$ of $\mathscr{P}$ is said to be upward directed if for each pair $x, y \in \mathscr{C}$ there is a $z \in \mathscr{C}$ such that $x \leq z$ and $y \leq z$. Similarly, $\mathscr{C}$ is downward directed if for each pair $x$, $y \in \mathscr{C}$ there is $w \in \mathscr{C}$ such that $w \leq x$ and $w \leq y$. If $\mathscr{C}$ is both upward and downward directed, it is called directed.

If there exist $u_{*}, u^{*} \in \mathcal{S}$ such that $u_{*} \leq u^{*}$, and $u_{*} \leq u \leq$ $u^{*}$, for any $u \in \mathcal{S}$, then $u_{*}$ and $u^{*}$ are called barrier solutions of (31) (see [4]). Apparently, $u_{*}$ and $u^{*}$ are the least element and the greatest element with respect to the ordering " $\leq$," respectively.

To show that $\mathcal{S}$ is directed with respect to the ordering " $\leq$," the following modulus of continuity condition on the coefficient $a_{1}: \Omega \times \mathbb{R} \times \mathbb{R}^{N} \longrightarrow \mathbb{R}^{N}$ is required:

$\left(A_{1}^{\prime}\right)$ For a.e. $x \in \Omega$, all $s, s^{\prime} \in \mathbb{R}$ and all $\xi \in \mathbb{R}^{N}$,

$$
\begin{aligned}
& \left|a_{1}(x, s, \xi)-a_{1}\left(x, s^{\prime}, \xi\right)\right| \\
& \leq\left[g_{3}(x)+\bar{\Phi}^{-1}(x, \Phi(x,|s|))+\bar{\Phi}^{-1}\left(x, \Phi\left(x,\left|s^{\prime}\right|\right)\right)\right. \\
& \left.\quad+\bar{\Phi}^{-1}(x, \Phi(x,|\xi|))\right] \omega\left(\left|s-s^{\prime}\right|\right),
\end{aligned}
$$

where $g_{3} \in E_{\bar{\Phi}}(\Omega), g_{3} \geq 0$, and $\omega: \mathbb{R}_{+} \longrightarrow \mathbb{R}_{+}$is a continuous function such that

$$
\int_{0}^{\varepsilon} \frac{1}{\omega(r)} d r=+\infty
$$

for every $\varepsilon>0$.

Theorem 8. Assume that the assumptions of Theorem 4 and $\left(A^{\prime}{ }_{1}\right)$ hold. Let $u_{1}, u_{2} \in \mathcal{S}$. Then $u_{1} \vee u_{2}$ and $u_{1} \wedge u_{2}$ are the subsolution and supersolution of (31), respectively.

Proof. Denote $V=W_{0}^{1} L_{\Phi}(\Omega)$. Let $u_{1}, u_{2} \in \mathcal{S}$. In view of assumption $\left(A^{\prime}{ }_{1}\right)$, for any $\varepsilon>0$, there exists $\delta(\varepsilon) \in(0, \varepsilon)$ such that $\int_{\delta(\varepsilon)}^{\varepsilon} 1 / \omega(r) d r=1$. Define the function $\theta_{\varepsilon}: \mathbb{R} \longrightarrow \mathbb{R}_{+}$, which is defined by

$$
\theta_{\varepsilon}(s)= \begin{cases}0, & \text { if } s<\delta(\varepsilon), \\ \int_{\delta(\varepsilon)}^{s} \frac{1}{\omega(r)} d r, & \text { if } \delta(\varepsilon) \leq s \leq \varepsilon \\ 1, & \text { if } s>\varepsilon\end{cases}
$$

Then, for every $\varepsilon>0$, the function $\theta_{\varepsilon}$ is continuous, piecewise differentiable, and the derivative is nonnegative and bounded. Consequently, $\theta_{\varepsilon}$ is Lipschitz continuous and nondecreasing, and it satisfies $\theta_{\varepsilon} \longrightarrow \chi_{\{s>0\}}$ as $\varepsilon \longrightarrow 0$. Moreover, $\theta_{\varepsilon}^{\prime}(s)=1 / \omega(s)$ if $\delta(\varepsilon) \leq s \leq \varepsilon$, and $\theta_{\varepsilon}^{\prime}(s)=0$ if $s \notin$ $[\delta(\varepsilon), \varepsilon]$ (see $[29])$.

Let $\psi \in V$. Replacing $u$ with $u_{1}, u_{2}$ and taking the test function $v=\psi\left(1-\theta_{\varepsilon}\left(u_{2}-u_{1}\right)\right), v=\psi \theta_{\varepsilon}\left(u_{2}-u_{1}\right)$ in (32), respectively, and adding the resulting equations, we obtain 


$$
\begin{aligned}
& \int_{\Omega} a_{1}\left(x, u_{1}, D u_{1}\right) D \psi d x \\
& \quad+\int_{\Omega}\left[a_{1}\left(x, u_{2}, D u_{2}\right)-a_{1}\left(x, u_{1}, D u_{1}\right)\right] D\left(\psi \theta_{\varepsilon}\left(u_{2}-u_{1}\right)\right) d x \\
& \quad+\int_{\Omega} a_{0}\left(x, u_{1}\right) \psi d x \\
& \quad+\int_{\Omega}\left[a_{0}\left(x, u_{2}\right)-a_{0}\left(x, u_{1}\right)\right] \psi \theta_{\varepsilon}\left(u_{2}-u_{1}\right) d x \\
& =\int_{\Omega} f\left(x, u_{1}, D u_{1}\right) \psi d x \\
& \quad+\int_{\Omega}\left[f\left(x, u_{2}, D u_{2}\right)-f\left(x, u_{1}, D u_{1}\right)\right] \psi \theta_{\varepsilon}\left(u_{2}-u_{1}\right) d x
\end{aligned}
$$
have

By applying (26), $\left(A^{\prime}{ }_{1}\right)$, and the properties of $\theta_{\varepsilon}$, we

$$
\begin{aligned}
& \int_{\Omega}\left[a_{1}\left(x, u_{2}, D u_{2}\right)-a_{1}\left(x, u_{1}, D u_{1}\right)\right] D\left(\psi \theta_{\varepsilon}\left(u_{2}-u_{1}\right)\right) d x \\
& =\int_{\Omega}\left[a_{1}\left(x, u_{2}, D u_{2}\right)-a_{1}\left(x, u_{1}, D u_{1}\right)\right] \theta_{\varepsilon}\left(u_{2}-u_{1}\right) D \psi d x \\
& \quad+\int_{\Omega}\left[a_{1}\left(x, u_{2}, D u_{2}\right)-a_{1}\left(x, u_{2}, D u_{1}\right)\right] \psi \theta_{\varepsilon}^{\prime}\left(u_{2}-u_{1}\right) D\left(u_{2}-u_{1}\right) d x \\
& \quad+\int_{\Omega}\left[a_{1}\left(x, u_{2}, D u_{1}\right)-a_{1}\left(x, u_{1}, D u_{1}\right)\right] \psi \theta_{\varepsilon}^{\prime}\left(u_{2}-u_{1}\right) D\left(u_{2}-u_{1}\right) d x \\
& \geq \int_{\Omega}\left[a_{1}\left(x, u_{2}, D u_{2}\right)-a_{1}\left(x, u_{1}, D u_{1}\right)\right] \theta_{\varepsilon}\left(u_{2}-u_{1}\right) D \psi d x \\
& \quad-\int_{\left\{\delta(\varepsilon)<u_{2}-u_{1}<\varepsilon\right\}}\left[g_{3}(x)+\bar{\Phi}^{-1}\left(x, \Phi\left(x,\left|u_{1}\right|\right)\right)\right. \\
& \left.\quad+\bar{\Phi}^{-1}\left(x, \Phi\left(x,\left|u_{2}\right|\right)\right)+\bar{\Phi}^{-1}\left(x, \Phi\left(x,\left|D u_{1}\right|\right)\right)\right]|\psi|\left|D\left(u_{2}-u_{1}\right)\right| d x
\end{aligned}
$$

The last term on the right-hand side of (60) tends to 0 , as $\varepsilon \longrightarrow 0$. Using Lebesgue's dominated convergence theorem, we obtain that

$$
\begin{gathered}
\int_{\Omega}\left[a_{1}\left(x, u_{2}, D u_{2}\right)-a_{1}\left(x, u_{1}, D u_{1}\right)\right] \theta_{\varepsilon}\left(u_{2}-u_{1}\right) D \psi d x \\
\longrightarrow \int_{\Omega}\left[a_{1}\left(x, u_{2}, D u_{2}\right)-a_{1}\left(x, u_{1}, D u_{1}\right)\right] \chi_{\left\{u_{2}>u_{1}\right\}} D \psi d x \\
\int_{\Omega}\left[a_{0}\left(x, u_{2}\right)-a_{0}\left(x, u_{1}\right)\right] \psi \theta_{\varepsilon}\left(u_{2}-u_{1}\right) d x \\
\quad \longrightarrow \int_{\Omega}\left[a_{0}\left(x, u_{2}\right)-a_{0}\left(x, u_{1}\right)\right] \chi_{\left\{u_{2}>u_{1}\right\}} \psi d x \\
\int_{\Omega}\left[f\left(x, u_{2}, D u_{2}\right)-f\left(x, u_{1}, D u_{1}\right)\right] \psi \theta_{\varepsilon}\left(u_{2}-u_{1}\right) d x \\
\longrightarrow \int_{\Omega}\left[f\left(x, u_{2}, D u_{2}\right)-f\left(x, u_{1}, D u_{1}\right)\right] \chi_{\left\{u_{2}>u_{1}\right\}} \psi d x
\end{gathered}
$$

as $\varepsilon \longrightarrow 0$.
Combining (59)-(63), we get that

$$
\begin{aligned}
& \int_{\Omega} a_{1}\left(x, u_{1}, D u_{1}\right) D \psi d x \\
& \quad+\int_{\Omega}\left[a_{1}\left(x, u_{2}, D u_{2}\right)-a_{1}\left(x, u_{1}, D u_{1}\right)\right] \chi_{\left\{u_{2}>u_{1}\right\}} D \psi d x \\
& \quad+\int_{\Omega} a_{0}\left(x, u_{1}\right) \psi d x+\int_{\Omega}\left[a_{0}\left(x, u_{2}\right)-a_{0}\left(x, u_{1}\right)\right] \chi_{\left\{u_{2}>u_{1}\right\}} \psi d x \\
& \leq \int_{\Omega} f\left(x, u_{1}, D u_{1}\right) \psi d x \\
& \quad+\int_{\Omega}\left[f\left(x, u_{2}, D u_{2}\right)-f\left(x, u_{1}, D u_{1}\right)\right] \chi_{\left\{u_{2}>u_{1}\right\}} \psi d x,
\end{aligned}
$$

which yields that $u_{1} \vee u_{2}$ is a subsolution of (31), i.e., $u_{1} \vee u_{2} \epsilon$ $\mathcal{S}$. Analogously, we can deduce that $u_{1} \wedge u_{2}$ is a supersolution of (31).

Theorem 9. Assume that the assumptions of Theorem 4 and $\left(A^{\prime}{ }_{1}\right)$ hold. Then, $\mathcal{S}$ is directed downward and upward; that is, for all $u_{1}, u_{2} \in \mathcal{S}$, there exist $u, v \in \mathcal{S}$ such that $v \leq u_{1} \wedge u_{2}$ and $u_{1} \vee u_{2} \leq u$.

Proof. Denote $V=W_{0}^{1} L_{\Phi}(\Omega)$. For $u_{1}, u_{2} \in \mathcal{S}$, let $u_{0}=u_{1} \vee u_{2}$. We will prove that there exists $\tilde{u} \in \mathcal{S}$ such that $u_{0} \leq \tilde{u}$.

Define the cutoff function $b: \Omega \times \mathbb{R} \longrightarrow \mathbb{R}$ by

$$
b(x, s)= \begin{cases}0, & \text { if } s>u_{0}(x) \\ -\bar{\Phi}^{-1}\left(x, \Phi\left(x, u_{0}(x)-s\right)\right), & \text { if } s \leq u_{0}(x)\end{cases}
$$

for $x \in \Omega, s \in \mathbb{R}$. Then for any $u \in L_{\Phi}(\Omega), b(x, u) \in L_{\bar{\Phi}}(\Omega)$.

Denote $\left\{u \leq u_{0}\right\}:=\left\{x \in \Omega: u(x) \leq u_{0}(x)\right\}$. For any $u \in V$, by Young inequality, one has

$$
\begin{aligned}
\int_{\Omega} b(x, u) u d x \geq & \int_{\left\{u \leq u_{0}\right\}} \Phi\left(x, u_{0}-u\right) d x \\
& -\int_{\left\{u \leq u_{0}\right\}} \frac{1}{2}\left[\Phi\left(x, u_{0}-u\right)+\Phi\left(x, 2\left|u_{0}\right|\right)\right] d x \\
\geq & -\frac{1}{2} \int_{\Omega} \Phi\left(x, 2\left|u_{0}\right|\right) d x
\end{aligned}
$$

Define $T u=u \vee u_{0}$. By Remark 3.1 in [1], $T: V \longrightarrow V$ is continuous. According to the definition of $T, T$ is bounded.

Thanks to (29), and by Young inequality, we have

$$
\begin{aligned}
\left|\int_{\Omega} F(T u) u d x\right| \leq & \left(2 b_{4}+b_{5}\right) \int_{\left\{u>u_{0}\right\}} \Phi(x,|u|) d x \\
& +b_{5} \int_{\Omega} \Phi(x,|D u|) d x+C_{1}\|u\|_{1, \Phi}
\end{aligned}
$$


for all $u \in V$, and some constant $C_{1}>0$ independent of $u$. From (27) and (28), we can deduce that

$$
\begin{aligned}
\int_{\Omega} a_{0}(x, T u) u d x \geq & b_{3} \int_{\left\{u>u_{0}\right\}} \Phi(x,|u|) d x \\
& -\int_{\left\{u>u_{0}\right\}} g_{2}(x) d x-C_{2}\|u\|_{1, \Phi},
\end{aligned}
$$

for all $u \in V$, and some constant $C_{2}>0$ independent of $u$.

Let us consider the following auxiliary equation of finding $u \in V$ such that

$$
\begin{aligned}
& \int_{\Omega} a_{1}(x, T u, D u) D v d x+\int_{\Omega} a_{0}(x, T u) v d x+\int_{\Omega} b(x, u) v d x \\
& \quad=\int_{\Omega} F(T u) v d x
\end{aligned}
$$

Define $\Gamma_{T}: V \longrightarrow V^{*}$,

$$
\begin{aligned}
\left(\Gamma_{T} u, v\right):= & \int_{\Omega} a_{1}(x, T u, D u) D v d x+\int_{\Omega} a_{0}(x, T u) v d x \\
& +\int_{\Omega} b(x, u) v d x-\int_{\Omega} F(T u) v d x
\end{aligned}
$$

$\forall v \in V$. Then, $\Gamma_{T}$ is well defined. have

Combining (25), (28), (29), (33), (66), (67), and (68), we

$$
\left(\Gamma_{T} u, u\right) \geq\left(b_{2}-b_{4}\right) \int_{\Omega} \Phi(x,|D u|) d x-C_{3}\|u\|_{1, \Phi}-C_{4},
$$
of $u$.

$\forall u \in V$, where $C_{3}, C_{4}>0$ are two constants independent

Similar to the proof of Theorem 4, we can deduce that there exists $\tilde{u} \in V$ such that

$$
\left(\Gamma_{T} \tilde{u}, v\right)=0, \quad \forall v \in V
$$

i.e., $\tilde{u}$ is a weak solution of (69).

Denote $\left\{u_{0}>\tilde{u}\right\}=\left\{x \in \Omega: u_{0}(x)>\tilde{u}(x)\right\}$. Replacing $u$ with $\tilde{u}$ in (69), and taking $v=\left(u_{0}-\tilde{u}\right)^{+} \in V$, we can obtain that

$$
\begin{aligned}
\int_{\Omega} a_{1}(x, T \tilde{u}, D \tilde{u}) D\left(u_{0}-\tilde{u}\right)^{+} d x \\
\quad+\int_{\Omega} a_{0}(x, T \tilde{u})\left(u_{0}-\tilde{u}\right)^{+} d x \\
\quad+\int_{\Omega} b(x, \tilde{u})\left(u_{0}-\tilde{u}\right)^{+} d x \\
=\int_{\Omega} F(T \tilde{u})\left(u_{0}-\tilde{u}\right)^{+} d x .
\end{aligned}
$$

By Theorem 8, we know

$$
\int_{\Omega} a_{1}\left(x, u_{0}, D u_{0}\right) D v d x+\int_{\Omega} a_{0}\left(x, u_{0}\right) v d x \leq \int_{\Omega} F\left(u_{0}\right) v d x,
$$

for any $v \in V$ with $v \geq 0$.

Taking $v=\left(u_{0}-\tilde{u}\right)^{+} \in V$ in (74), we have

$$
\begin{aligned}
& \int_{\Omega} a_{1}\left(x, u_{0}, D u_{0}\right) D\left(u_{0}-\tilde{u}\right)^{+} d x+\int_{\Omega} a_{0}\left(x, u_{0}\right)\left(u_{0}-\tilde{u}\right)^{+} d x \\
& \quad \leq \int_{\Omega} F\left(u_{0}\right)\left(u_{0}-\tilde{u}\right)^{+} d x .
\end{aligned}
$$

Subtracting (73) from (75), we obtain that

$$
\begin{aligned}
\int_{\Omega}[ & \left.a_{1}\left(x, u_{0}, D u_{0}\right)-a_{1}(x, T \tilde{u}, D \tilde{u})\right] D\left(u_{0}-\tilde{u}\right)^{+} d x \\
& +\int_{\Omega}\left[a_{0}\left(x, u_{0}\right)-a_{0}(x, T \tilde{u})\right]\left(u_{0}-\tilde{u}\right)^{+} d x \\
& -\int_{\Omega} b(x, \tilde{u})\left(u_{0}-\tilde{u}\right)^{+} d x \\
\leq & \int_{\Omega}\left[F\left(u_{0}\right)-F(T \tilde{u})\right]\left(u_{0}-\tilde{u}\right)^{+} d x .
\end{aligned}
$$

Thanks to (26), the first term of the left-hand side of (76) is nonnegative. Clearly, both the second term of the lefthand side and the right-hand side of (76) equal 0 . Indeed,

$$
\begin{aligned}
& \int_{\Omega}\left[a_{0}\left(x, u_{0}\right)-a_{0}(x, T \tilde{u})\right]\left(u_{0}-\tilde{u}\right)^{+} d x \\
& \quad=\int_{\left\{u_{0}>\tilde{u}\right\}}\left[a_{0}\left(x, u_{0}\right)-a_{0}\left(x, u_{0}\right)\right]\left(u_{0}-\tilde{u}\right) d x=0, \\
& \int_{\Omega}\left[F\left(u_{0}\right)-F(T \tilde{u})\right]\left(u_{0}-\tilde{u}\right)^{+} d x \\
& =\int_{\left\{u_{0}>\tilde{u}\right\}}\left[F\left(u_{0}\right)-F\left(u_{0}\right)\right]\left(u_{0}-\tilde{u}\right) d x=0 .
\end{aligned}
$$

Therefore,

$$
\int_{\Omega} b(x, \tilde{u})\left(u_{0}-\tilde{u}\right)^{+} d x \geq 0
$$

On the other hand,

$$
\begin{aligned}
\int_{\Omega} b(x, \tilde{u})\left(u_{0}-\tilde{u}\right)^{+} d x & =-\int_{\left\{u_{0}>\tilde{u}\right\}} \bar{\Phi}^{-1}\left(x, \Phi\left(x, u_{0}-\tilde{u}\right)\right)\left(u_{0}-\tilde{u}\right) d x \\
& \leq-\int_{\left\{u_{0}>\tilde{u}\right\}} \Phi\left(x, u_{0}-\tilde{u}\right) d x \leq 0 .
\end{aligned}
$$

It follows that $u_{0} \leq \tilde{u}$. 
From the definitions of $l, T$, we deduce that

$$
\begin{aligned}
& a_{1}(x, T \tilde{u}(x), D \tilde{u}(x))=a_{1}(x, \tilde{u}(x), D \tilde{u}(x)), \\
& a_{0}(x, T \tilde{u}(x))=a_{0}(x, \tilde{u}(x)), \\
& b(x, \tilde{u}(x))=0, f(x, T \tilde{u}(x), D T \tilde{u}(x))=f(x, \tilde{u}(x), D \tilde{u}(x)),
\end{aligned}
$$

for a.e. $x \in \Omega$. We note that (69) reduces to (32). Hence, $\tilde{u}$ is a weak solution of (31). Consequently, $\tilde{u} \in \mathcal{S}$, and $u_{1} \vee u_{2} \leq \tilde{u}$.

Similarly, there exists $v \in \mathcal{S}$, such that $v \leq u_{1} \wedge u_{2}$. Therefore, $\mathcal{S}$ is a directed set.

Similar to step (c) of the proof of Theorem 3.1 in [4], we have the following result.

Theorem 10. Assume that the assumptions of Theorem 4 and $\left(A_{1}^{\prime}\right)$ hold. Let $\Phi \in \Delta_{2}$. Then (31) admits barrier solutions, i.e., there exist $u_{*}, u^{*} \in \mathcal{S}$ such that $u_{*} \leq u \leq u^{*}, \forall u \in \mathcal{S}$.

\section{Data Availability}

No data were used to support this study.

\section{Conflicts of Interest}

The authors declare that there are no conflicts of interest regarding the publication of this paper.

\section{Acknowledgments}

The first author was supported by the 100 Teachers Database Project of Shanghai University of Medicine and Health Sciences (B30200203110084) and the Mandatory Plan Project of SUMHS (CZ401). The second author was supported by the National Natural Science Foundation of China (11871375).

\section{References}

[1] X. L. Fan, "Differential equations of divergence form in Musielak-Sobolev spaces and a sub-supersolution method," Journal of Mathematical Analysis and Applications, vol. 386, no. 2, pp. 593-604, 2012.

[2] G. Dong and X. C. Fang, "Differential equations of divergence form in separable Musielak-Orlicz-Sobolev spaces," Boundary Value Problems, vol. 2016, no. 1, Article ID 106, p. 19, 2016.

[3] S. Carl and V. K. Le, "Elliptic inequalities with multi-valued operators: existence, comparison and related variationalhemivariational type inequalities," Nonlinear Analysis: Theory, Methods \& Applications, vol. 121, pp. 130-152, 2015.

[4] S. Carl, "Barrier solutions of elliptic variational inequalities," Nonlinear Analysis: Real World Applications, vol. 26, pp. 7592, 2015.

[5] M. Chipot, S. Guesmia, and S. Harkat, "On the minimal solution for some variational inequalities," Journal of Differential Equations, vol. 266, no. 1, pp. 493-525, 2019.

[6] P. Gwiazda, I. Skrzypczak, and A. Zatorska-Goldstein, "Existence of renormalized solutions to elliptic equation in Musielak-Orlicz space," Journal of Differential Equations, vol. 264, no. 1, pp. 341-377, 2018.
[7] I. Chlebicka and A. Karppinen, "Removable sets in elliptic equations with Musielak-Orlicz growth," Journal of Mathematical Analysis and Applications, vol. 501, no. 1, article 124073, 2021.

[8] V. K. Le, "On a sub-supersolution method for variational inequalities with Leray-Lions operators in variable exponent spaces," Nonlinear Analysis: Theory, Methods \& Applications, vol. 71, no. 7-8, pp. 3305-3321, 2009.

[9] L. F. O. Faria, O. H. Miyagaki, D. Motreanu, and M. Tanaka, "Existence results for nonlinear elliptic equations with LerayLions operator and dependence on the gradient," Nonlinear Analysis: Theory, Methods \& Applications, vol. 96, pp. 154166, 2014.

[10] J. Liu, P. Pucci, H. Wu, and Q. Zhang, "Existence and blow-up rate of large solutions of $\mathrm{p}(\mathrm{x})$-Laplacian equations with gradient terms," Journal of Mathematical Analysis and Applications, vol. 457, no. 1, pp. 944-977, 2018.

[11] J. Gossez, "Nonlinear elliptic boundary value problems for equations with rapidly (or slowly) increasing coefficients," Transactions of the American Mathematical Society, vol. 190, pp. 163-205, 1974.

[12] J. Gossez, "Some approximation properties in Orlicz-Sobolev spaces," Studia Mathematica, vol. 74, no. 1, pp. 17-24, 1982.

[13] G. Dong and Z. R. Shi, "An existence theorem for weak solutions for a class of elliptic partial differential systems in Orlicz spaces," Nonlinear Analysis: Theory, Methods \& Applications, vol. 68, no. 4, pp. 1037-1042, 2008.

[14] G. Dong, “An existence theorem for weak solutions for a class of elliptic partial differential systems in general Orlicz-Sobolev spaces," Nonlinear Analysis: Theory, Methods \& Applications, vol. 69, no. 7, pp. 2049-2057, 2008.

[15] G. Dong, "Elliptic equations with measure data in Orlicz spaces," Electronic Journal of Differential Equations, vol. 76, pp. 1-10, 2008.

[16] G. Dong and X. C. Fang, "Variational inequalities with multivalued lower order terms and convex functionals in OrliczSobolev spaces," Journal of Function Spaces, vol. 2015, Article ID 321437, 10 pages, 2015.

[17] G. Dong and X. C. Fang, "The sub-supersolution method and extremal solutions of quasilinear elliptic equations in OrliczSobolev spaces," Journal of Function Spaces, vol. 2018, Article ID 8104901, 7 pages, 2018.

[18] G. Dong and X. C. Fang, "Positive solutions to nonlinear inclusion problems in Orlicz-Sobolev spaces," Applicable Analysis, vol. 100, no. 7, pp. 1440-1453, 2021.

[19] Z. Bashir, J. L. G. Guirao, A. Siddique, and T. Saeed, "Generalized concentration-compactness principles for variable exponent Lebesgue spaces with asymptotic analysis of low energy extremals," Mathematics, vol. 8, no. 10, article 1849, 2020.

[20] P. Chen and X. Tang, "Periodic solutions for a differential inclusion problem involving the $\mathrm{p}(\mathrm{t})$-Laplacian," Advances in Nonlinear Analysis, vol. 10, no. 1, pp. 799-815, 2021.

[21] G. Lu, "Commutators of bilinear pseudo-differential operators on local Hardy spaces with variable exponents," Bulletin of the Brazilian Mathematical Society, vol. 51, no. 4, pp. 975-1000, 2020.

[22] M. A. Ragusa and A. Tachikawa, "On interior regularity of minimizers of $\mathrm{p}(\mathrm{x})$-energy functionals," Nonlinear Analysis: Theory, Methods \& Applications, vol. 93, pp. 162-167, 2013.

[23] J. Musielak, Orlicz Spaces and Modular Spaces, Lecture Notes in Math, Springer-Verlag, Berlin, 1983. 
[24] C. Wu, T. Wang, S. Chen, and Y. Wang, Geometry Theory of Orlicz Spaces, Harbin University of Technology Press, Harbin, 1986.

[25] A. Benkirane and M. Sidi El Vally, "Variational inequalities in Musielak-Orlicz-Sobolev spaces," Bulletin of the Belgian Mathematical Society, Simon Stevin, vol. 21, no. 5, pp. 787-811, 2014.

[26] J. Rodrigues and R. Teymurazyan, "On the two obstacles problem in Orlicz-Sobolev spaces and applications," Complex Variables and Elliptic Equations, vol. 56, no. 7-9, pp. 769787, 2011.

[27] G. Dong and X. C. Fang, "Existence results for some nonlinear elliptic equations with measure data in Orlicz-Sobolev spaces," Boundary Value Problems, vol. 2015, no. 1, Article ID 18, 2015.

[28] A. Benkirane and M. O. M. Val, "Some approximation properties in Musielak-Orlicz-Sobolev spaces," Thai Journal of Mathematics, vol. 10, no. 2, pp. 371-381, 2012.

[29] S. Carl, V. K. Le, and D. Motreanu, Nonsmooth Variational Problems and Their Inequalitiess: Comparison Principles and Applications, Springer Monographs in Mathematics, Springer, New York, NY, USA, 2007. 